\title{
Making Snakes Converge from Minimal Initialization
}

\author{
W. Neuenschwander*, P. Fua ${ }^{\dagger}$, G. Székely* and O. Kübler* \\ * Communication Technology Laboratory \\ $\dagger$ SRI International
Artificial Intelligence Center \\ Swiss Federal Institute of Technology \\ ETH \\ CH-8092 Zurich, Switzerland \\ 333 Ravenswood Avenue \\ Menlo Park, CA 94025, USA
}

\begin{abstract}
In this paper, we present a new snake-based method to delineate contours where the user has to specify only the two distant end points without having to supply an almost complete polygonal approximation. We achieve much better convergence properties than those of traditional snakes by propagating the image information along the curve from both end points towards its center. We use our new method to outline curved object boundaries and for interactive road delineation.
\end{abstract}

\section{Introduction}

Conceptual elegance and flexible, dynamic behavior have made snakes emerge as a very powerful tool for semiautomated object delineation. They have been originated by Terzopoulos, Kass, and Witkin $[1,2]$ and have since given rise to a large body of literature ( $[3,4,5]$ among many others). The appeal of active contour models has sometimes obscured the fact that snakes can be treacherous. Often the dynamic evolution from an initial configuration to a desired target image structure is ill natured and converges to questionable final states.

The optimization of the traditional snakes is typically global and takes edge-information into account along the whole curve simultaneously. When the snake's initial position is far away from the desired result, this often results in the snake getting stuck in an undesirable local minimum because it uses irrelevant edge information.

Our snake approach allows a user to specify only the end points of the curve he wishes to delineate instead of a complete polygonal approximation. The optimization progresses from the end points towards the center thereby effectively propagating edgeinformation along the curve. As a result, the snake eventually finds the smooth path, as defined by the regularization term, that best matches the edge connecting the two end points and has the right orienta- tion at these points.

Our efforts are aimed at alleviating the often repetitive task practitioners have to face when segmenting images. This way we may abolish the need to outline the desired structure very precisely, that is, to perform a painstaking, almost complete, manual segmentation.

\section{Mathematical snake formalism}

The original snakes [2] are modeled as time dependent 2-D curves defined parametrically as $\vec{v}(s, t)=$ $(x(s, t), y(s, t))^{T}$ where $s$ is proportional to the arc length, $t$ the current time and $x$ and $y$ the curve's image coordinates. The snake deforms itself as time progresses so as to minimize an image potential $E_{I}(\vec{v})=$ $-\int_{0}^{1} P(\vec{v}(s, t)) d s$, where $P(\vec{v}(s, t))$ is a function of the image, typically the magnitude of the image gradient.

To perform the optimization, following Terzopoulos et al., one must minimize an energy $E(\vec{v})$ that is the sum of $E_{I}(\vec{v})$ and of a regularization term $E_{D}(\vec{v})$. Using the thin-plate model, $E_{D}(\vec{v})$ is taken to be

$$
E_{D}(\vec{v})=\frac{1}{2} \int_{0}^{1} \alpha(s)\left|\frac{\partial \vec{v}(s, t)}{\partial s}\right|^{2}+\beta(s)\left|\frac{\partial^{2} \vec{v}(s, t)}{\partial s^{2}}\right|^{2} d s,
$$

where $\alpha(s)$ and $\beta(s)$ are arbitrary functions that regulate the curve's tension and rigidity. In our implementation, $\alpha$ and $\beta$ are taken to be constant and can be chosen in a fairly image-independent way [3]. Several techniques, however, have been proposed to dynamically adjust the values of $\alpha, \beta$ along the curve (e.g. [6]).

Following [3] we have also implemented "ribbon snakes" as a tool for interactive road delineation. The model vector $\vec{v}$ is augmented by a third component, the varying width of the road. The expression for the deformation energy (1) still holds for $\vec{v}(s, t)=$ $(x(s, t), y(s, t), w(s, t))^{T}$, where the width is subject to tension and rigidity constraints like the two coordinate components. The ribbon forms the center of the road while the assigned width defines two curves which are the actual deforming road boundaries. The 
(a)

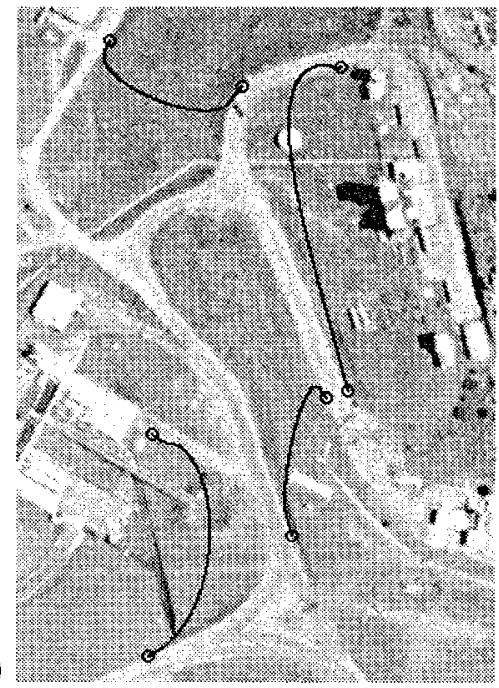

(b)

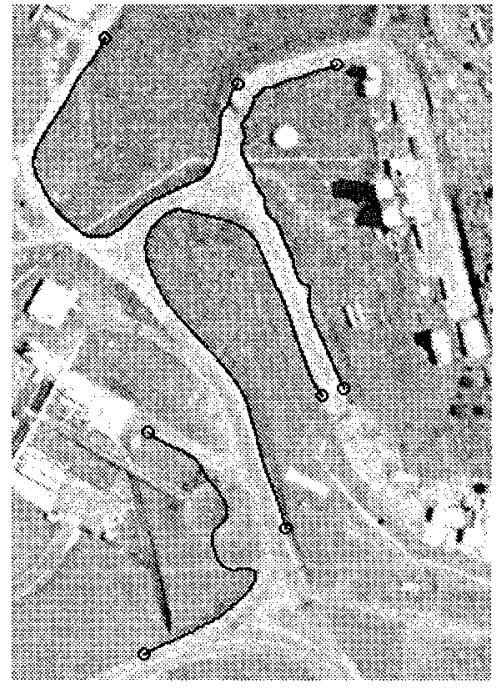

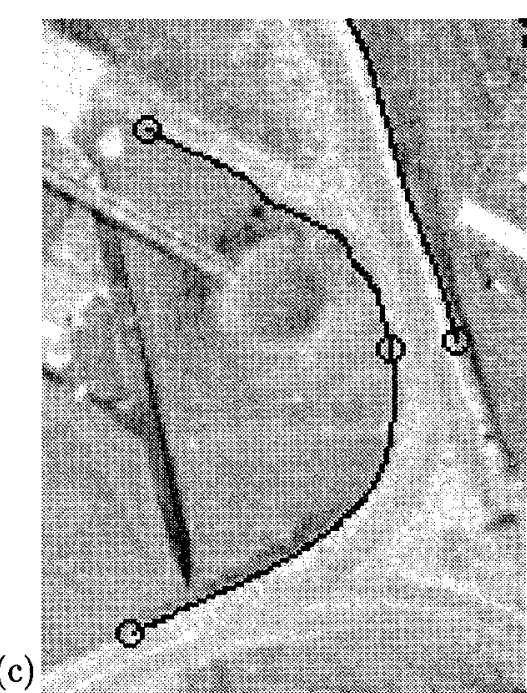

Figure 1: Outlining roads in an aerial image. (a) Snake initializations (unique solutions of the homogeneous Euler DE).

(b) Final results. All the road edges are correctly outlined except the bottom left one. (c) The erroneous result is corrected by adding a new control point in the middle of the curve, thereby splitting it into two snakes.

image information is taken into account along these two curves only.

\section{Optimization scheme}

Minimizing (1) is equivalent to solving the fourth order Euler differential equation

$$
-\alpha \frac{\partial^{2}}{\partial s^{2}} \vec{v}(s)+\beta \frac{\partial^{4}}{\partial s^{4}} \vec{v}(s)=-\nabla P(\vec{v}(s))
$$

We therefore start the optimization of the energy term by defining the initial snake as the solution of the homogeneous differential equation.

The discretization of the total energy and the equation (2) yield both the same singular, penta-diagonal stiffness matrix $K$ which defines a system of linear equations [7].

Equation (2) is of fourth order and therefore requires 4 boundary values for a unique solution. In our implementation, we assume that the user specifies snake end points in the vicinity of clearly visible edge segments, which implies a well defined edge direction. By supplying the locations of the two end points and the associated edge directions we can compute a unique initial snake which lies already close to its optimal position at both ends. Note, this process yields a modified stiffness matrix $K^{\prime}$ which has become regular and is therefore invertible [7].

At this stage the snake "feels" absolutely no external potential forces. During the ongoing iterative optimization process the image potential is turned on progressively for all the snake vertices, starting from the extremities. We define the "force boundaries" as the location of the vertices farthest away from the end points that feel the image forces. These boundaries approach each other during the ongoing optimization process by moving forward at every iteration step if the displacement between the last two iterations is below a certain threshold.

By iterating this process, we eventually turn on the image forces over the whole length of the snake, thereby achieving the propagation of the edge information from the anchor points to the snake's middle.

\section{Results}

In figure 1 we demonstrate that our snakes can be used to delineate roads in an aerial image using very

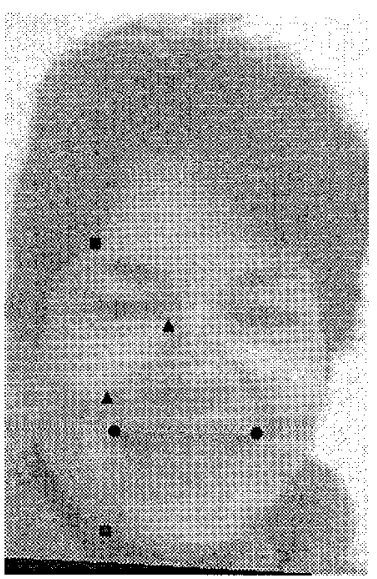

(a)

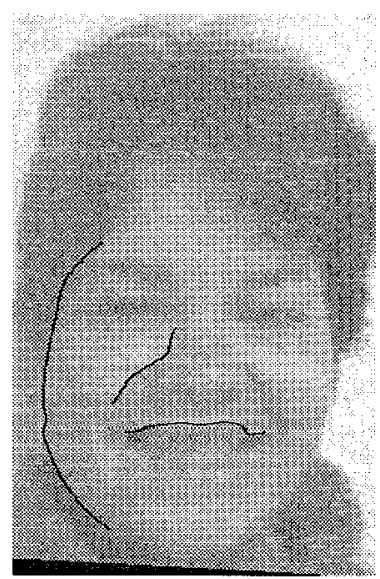

(b)
Figure 3: Outlining facial features. (a) Three pairs of end points on a face image (Courtesy of INRIA). (b) Final results. 
(a)

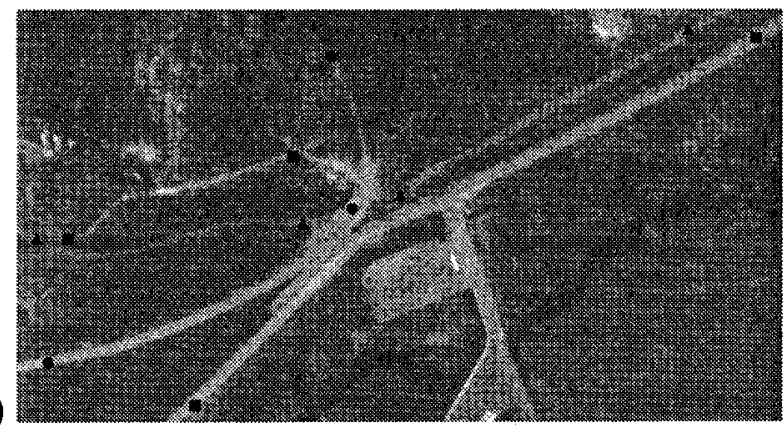

(b)

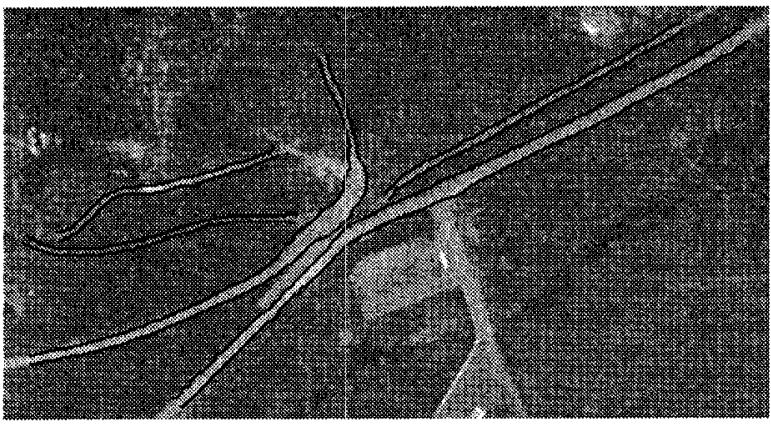

Figure 2: Delineating roads on an aerial image using ribbon snakes. (a) Several pairs of end points. (b) Final deteced road segments. Note, that the actual ribbon, the centerline of the road, is not shown in the right-hand side image.

distant end-points. Note, however, that our snakes can still become confused in the presence of junctions.

Figure 3 shows the snake's performance on a low contrast face image. These results illustrate our snake model's capabilty to leave potential valleys and close small gaps.

Figure 2 depicts the delineation of complete road boundaries using the ribbon snake model. Each ribbon is initialized by the two corresponding end points and the road direction which is the average direction of the left and right road boundary.

We have implemented an interactive tool for the segmentation of more complex shapes. By defining subsequently end points for adjoining open snake fragments the initial segment is extended under immediate visual control in a sort of contour-following process. Figure 4 illustrates this process on the segmentation of a corpus callosum in an MR image.
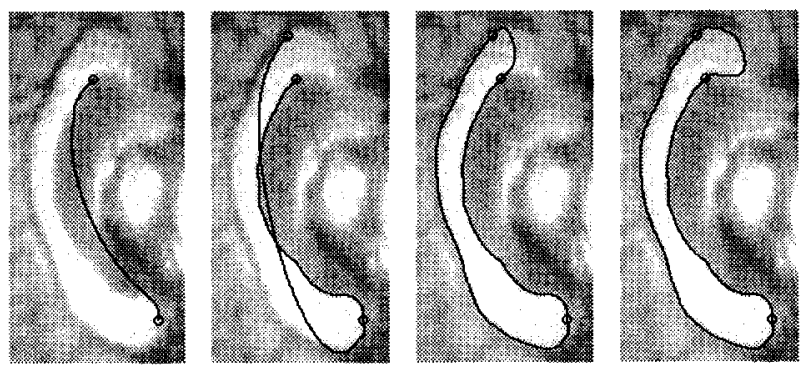

Figure 4: Segmentation process of a corpus callosum (MR image slice turned by $90^{\circ}$ ). The closed contour is built up continually by 3 open snakes linked by the 3 fix points (o).

\section{Conclusion}

We have proposed a snake-based approach to semiautomated delineation that allows a user to outline an open contour by only specifying very distant end points and allowing the computer to propagate edgeinformation from the extremities towards the center. This yields excellent convergence properties for the snakes and substantially reduces the probability of getting trapped into an undesirable local minimum.
By sequentially defining end points of adjoining open snake fragments we are able to segment more complex shapes. Specifying a discrete set of points along the contour thus becomes the natural initialization procedure completely in line with the practitioner's task.

We have extended our method to ribbon snakes that can be used to easily delineate complete road boundaries.

Intelligent initialization of snakes is required to make them into an operational tool for image segmentation where existing implementations leave almost all the work to an "expert user" [2]. Our method has been designed for practitioners of image evaluation without a professional background in image understanding.

We will concentrate our future research on generalizing our approach to handle more general constraints and problems of higher dimensionality.

\section{References}

[1] D. Terzopoulos, A. Witkin, and M. Kass. Symmetry-seeking Models for 3D Object Reconstruction. IJCV, 1(3):211-221, October 1987.

[2] M. Kass, A. Witkin, and D. Terzopoulos. Snakes: Active contour models. IJCV,1(4):321-331,1988.

[3] P. Fua and Y.G. Leclerc. Model Driven Edge Detection. Machine Vision and Applications, 3:45$56,1990$.

[4] I. Cohen, L. D. Cohen, and N. Ayache. Using Deformable Surfaces to Segment 3-D Images and Infer Differential Strucutres. CVGIP: IU, 56(2):242263, September 1992.

[5] F. Leymarie and M.D. Levine. Tracking deformable objects in the plane using an active contour model. IEEE PAMI, 15(6):617-634, 1993.

[6] R. Samadani. Changes in connectivity in active contour models. In Proceedings of the IEEE Workshop on Visual Motion, Irvine, California, pages 337-343, March 1989.

[7] W. Neuenschwander, P. Fua, G. Székely and O. Kübler. Initializing Snakes. In $C V P R$, pages 658663, Seattle, WA, 1994 